\title{
Is a "Decentralized Autonomous Organization" a Panopticon?
}

\author{
Algorithmic governance as creating and mitigating vulnerabilities in DAOs \\ Kelsie Nabben \\ Blockchain Innovation Hub \\ RMIT University \\ Melbourne, VIC, Australia \\ kelsie.nabben@,rmit.edu.au
}

\begin{abstract}
This piece explores algorithmic governance as a strength and a vulnerability in the experience of building participatory communities known as "Decentralized Autonomous Organizations". The Cypherpunks were terrified of surveillance. They envisaged the combination of cryptography and computer technology fundamentally altering the nature of trust and reputation and built cryptographically secure blockchain-based infrastructure to counter this threat. Now, not just on chain transactions are being tracked but every move of participants in blockchain communities. Reputation in blockchain systems could become the new algorithmic authoritarianism if mis-used for social control. This piece analyzes the ways in which decentralization efforts can be a threat to themselves by exploring the question, "Are "Decentralized Autonomous Organizations" (DAOs) the next panopticon of algorithmic governance or a different panacea, and what does this mean for human autonomy in "autonomous" systems?'. By employing ethnographic methods and case study analysis, this piece provides an important qualitative contribution to the early dynamics of the aspirations and problems of decentralized, autonomous organizations.
\end{abstract}

\section{CCS CONCEPTS}

- Security and privacy $\rightarrow$ Human and societal aspect of security and privacy $\bullet$ Applied computing Law, social and behavioral sciences $\rightarrow$ Anthropology $\rightarrow$

Ethnography $\bullet$ Computer systems organization $\rightarrow$ Peer-to-peer architectures.

\section{KEYWORDS}

Algorithmic governance, Decentralized Autonomous Organization, blockchain

\section{ACM Reference format:}

Kelsie Nabben. 2021. Is a "Decentralized Autonomous Organization" a Panopticon? Algorithmic governance as creating and mitigating vulnerabilities in DAOs. In Interdisciplinary Workshop on (de) Centralization in the Internet (IWCT'21), December 7, 2021. Virtual Event, Germany. ACM, New York, NY, USA, 8 pages. https://doi.org/10.1145/3488663.3493791
Permission to make digital or hard copies of all or part of this work for personal or classroom use is granted without fee provided that copies are not made or distributed for profit or commercial advantage and that copies bear this notice and the full citation on the first page. Copyrights for components of this work owned by others than the author(s) must be honored. Abstracting with credit is permitted. To copy otherwise, or republish, to post on servers or to redistribute to lists, requires prior specific permission and/or a fee. Request permissions from permissions@acm.org.

IWCI '21, December 7, 2021, Virtual Event, Germany

(C) 2021 Copyright held by the owner/author(s). Publication rights licensed to the Association for Computing Machinery.

ACM ISBN 978-1-4503-9138-2/21/12.

https://doi.org/10.1145/3488663.3493791

\section{Introduction}

The ultimate instantiation of the value of political decentralization is autonomy. This is uniquely expressed in decentralized technology communities in the idea of "Decentralized Autonomous Organizations" (DAOs) as a novel institutional structure for collective governance through technology. DAOs promise "autonomy" in terms of independence and selfgovernance through decentralized technology systems [1]. DAOs are automated decision-making systems (ADMs), defined as a system, software, or process that uses computation to aid or replace government decisions, judgements, and/or policy implementation that can involve predicting, classifying, optimizing, identifying, and/or recommending, to impact opportunities, access, liberties, rights, and/or safety [2]. What is being automated for autonomous coordination in these organizations is some level of trust [3]. Jasanoff and Kim label these kinds of collective visions of the future implicated in technology as "sociotechnical imaginaries" [4]. Blockchains are deeply implicated with imaginaries of freedom, liberty, and selfgovernance through technologically coordinated social and economic systems [5].

The panopticon is an $18^{\text {th }}$ Century thought experiment by philosopher Jeremy Bentham about how to create the ultimate prison [6]. Bentham envisaged a circular prison with an inspection tower in the middle that could see over everything, yet prisoners could not see into it to know if it was attended and if they were being surveilled [6]. Thus, prisoners are subject to the insecurity that they are always being monitored by the omnipotent authority. The result of the constant assumption of surveillance is behavior 
medication, to the point where prisoners monitor each other's behavior to obey the rules and avoid punishment out of fear of surveillance. The ultimate consequence of the Panopticon is that inmates is that they modify their own behavior and police themselves, although the tower could be empty. Scholars have adopted the concept of the Panopticon to describe digital surveillance in current internet platforms and applications [7].

Threat analysis in blockchains usually focuses on explicit, exogenous attacks, those originating outside of the organism, such as a DAO itself [8]. In this piece, I also explore implicit, endogenous vulnerabilities, which are threats that emerge from within the institutional structure of the DAO. Implicit vulnerabilities both challenge stability and create opportunities for the DAO to adapt and evolve to become more resilient. Through a case study analysis, I explore the interplay between "sybil attacks", when an attacker subverts the reputation system of a network service by creating a large number of pseudonymous identities to exploit the system, and algorithmically managed "reputation" systems as a mechanism to solve trust and coordinate as a geographically distributed community at scale. Yet, algorithmic aggregation and enforcement of reputation is also a highly efficient mechanism of social control that is antithetical to the public blockchain community values of political decentralization and autonomy [9]. So, are reputation mechanisms used in blockchain communities to realize the autonomy of participants, or as a highly efficient mechanisms of algorithmic social control, and what does this mean for people's autonomy and resilience in "autonomous" systems?

This piece demonstrates how algorithmic governance both addresses and creates vulnerabilities in decentralized systems. I argue that humans and algorithms are co-constitutive, and the difference between algorithmic governance in DAOs and existing digital platforms is that creation and amendment of the rules is participatory in nature. I then analyze how blockchain communities respond and engage with algorithms with a sense of agency by shaping algorithmic rules through a culture of participation to demonstrate that the greatest threat to a DAO is itself in its culture, ideology, and algorithmic processes that are defined by its participants. I argue that the adaptability of these participants is also its greatest strength towards resilience.

The methods employed are digital ethnography and case study analysis. This analysis provides a significant contribution to thinking about the human outcomes of algorithmic governance as it relates to the idea of who, or what, is being made "autonomous" in digital infrastructures, and how resilience is outworked in socio-technical institutions.

\section{The algorithmic governance of reputation in blockchain systems}

The term DAO stands for "Decentralized Autonomous Organization", and refers to a physically distributed group of people, participating in a shared goal. This coordination function is often enacted by digital tools, although it is both social and technical. DAOs try to be "decentralized" by leveraging public, decentralized blockchains as the base layer on which they operate for governance processes (such as "on-chain" voting) and exchange of value (such as payment for labor in cryptocurrency).

Blockchain practitioners often recognize possible external attacks such as sybil attacks as a dominant threat to DAOs. A sybil attack is when an attacker subverts the reputation system of a network service by creating multiple pseudonymous identities to exploit the system, and is often done computationally, using algorithmically programmed bots [10]. Algorithmically automated reputation management is a core tenant of decentralized system design to protect against "sybil attacks". Reputation mechanisms such as "praise", "cred", and "trust scores" are common tools by DAOs to verify participant identity to establish a basis of trust in the community. A crucial attribute that decentralized technology communities need to solve in order to function as scalable, autonomous, trust coordination machines is decentralized, digital reputation.

Reputation is a socially formed opinion about a person or entity. It is used to establish and enforce social norms of behavior for a community to interact and function according to these shared understandings and rules. In blockchains, reputation accrues political power [11]. Reputation is a currency of legitimacy which is fundamental to maintaining anonymous trust to coordinate in decentralized networks. In these communities, some actors choose to be only represented by a pseudonym or a public key address. As such, their previous behaviors as judged by peers in the community are the most viable predictor of future behaviors, rather than a name, endorsement, or resume. In these communities, reputation is all you have. It is also a mechanism of governance [12].

To manage reputation in digitally mediated, decentralized communities, reputation is being measured and enforced by algorithms that monitor, aggregate, and reward what has been deemed as desirable behavior in accordance with the norms of each community. Already, a significant amount of information about user behaviors is transparent and publicly available on the open ledger of public blockchain transaction data to be analyzed by law enforcement, data analytics companies, and peers in the network [13]. Now, reputation and reward in Decentralized Autonomous Organizations goes beyond what is known by about a public key address associated with on chain transaction data to algorithmically monitor behavior and assess contributions of attention, time, and labor in a DAO. Algorithmic governance in blockchain communities has been referred to as "governance by algorithms" [14]. The algorithmic rules of the system dictate incentives and determine rewards in what could be conceived of as a highly efficient mechanism of behavior direction and social control. 
The goal of algorithmic games in decentralized systems is scalable coordination: to scale trust in social institutions without a central intermediary. When algorithms are deployed as operators or agents in the system and rules and decisions are automatically carried out by smart contracts, the notion of "autonomous", selfgoverning systems emerges [15]. Autonomy is a binding imaginary of blockchain communities, which was envisaged as individual freedom through self-governance, and is threatened by the pervasion of algorithmic reputation and reward systems.

\section{History: the dream of freedom through pseudonymous reputation}

The "Cypherpunks" were hackers that contributed key ideas and technologies that led to the invention of Bitcoin. "Reputations will be of central importance, far more important in dealings than even the credit ratings of today" notes Timothy C. May, co-founder of the Cypherpunk's Mailing List in the "Crypto Anarchist Manifesto [16]. As early as 1994, the Cypherpunks envisaged cyberspacial "virtual network communities" that would profoundly alter the nature of economic and social systems, comprised of public key cryptography, secure e-mailing, digital cash, and reputation-based voting systems [17]. Their goal was to develop anonymous, untraceable systems for autonomy - as freedom from external surveillance or coercion in private affairs. According to the Cypherpunks, "virtual communities are the networks of individuals or groups which are not necessarily closely-connected geographically" [18]. Blockchain communities have largely embraced this ideology and are actively developing the tools to enable multiverses of autonomy-enabling digital realities. Yet, to solve the governance challenges of determining reputation and rewards, DAOs are aggressively embracing algorithmic surveillance. This includes attention monitoring on forums, "trust" scores through the provision of personal data, and requiring participation be proven before rewards are issued.

\subsection{Algorithms everywhere}

Algorithmic reputation is not new in our everyday lives. We are already being "algorithmically governed" through the of major social media and search platforms [19]. People are used to checking a seller's eBay percentage score before purchasing an item, the beautiful blue "tick" next to a known persona's Twitter handle, or the academic "H-index" score of on Google Scholar that determines your next role. Good reputation results in economic transactions and is good for business. We have become accustomed to this process being algorithmically determined for us. Search engines and social media platforms (such as Google, Facebook and Instagram) prioritize and serve up content that performs well over time to maintain user engagement and increase advertising revenues [20]. We know these tactics of algorithmic governance are influencing and controlling our behaviors behind the "black box" veil of closed-source software code, although we don't mind because of the efficiency and convenience these services. The epitome of a fully autonomous digitally enabled social system is often referred to as China's Social Credit System (SCS), "that treats government as nothing more than a great computer" [21]. The SCS prioritizes economic efficiency and political control by seeking to "prevent behavior deemed undesirable" and "encourage behavior deemed desirable" [22]. Algorithms are being employed for the same purposes to guide politics and economics in blockchain-based systems.

\subsection{Algorithms are subjective}

Social scientists argue that is important to look not just at algorithms and how they seek to influence and dictate behaviors but also to observe how everyday users respond. Empirical analyses of everyday encounters with algorithms help to contextualize the realities of algorithmic power. In "Atlas of an AI", Crawford argues that algorithms are neither autonomous, nor rational but rather are a product of extensive training of large datasets and pre-determined rules and rules, designed to extract value from infrastructure, capital, and labor [23]. Studies of social media platforms observe that platform operators hold a significant degree of power in determining the institutional conditions of user behavior and labor within platforms, although subjects will often adopt tactics to evade their power [24]. This is analogous to the example of the soon-to-be DAO blockchain game "Axie Infinity". This reputation-based blockchain game is leveraging attention in a "Play-to-Earn" model which has seen widespread adoption in Filipino communities [25]. The game allows players to breed, raise, and battle digital creatures called "Axies". Players earn reputation in the form of experience points for the time they play. The scarcity of Axies that are bred is determined by sophisticated "cryptoeconomics" called a "token bonding curve" [26]. Entire villages in Southeast Asia are being incentivized to play around the clock to earn around $\$ 200$ - $\$ 400$ a week. While this provides access to income perhaps not otherwise available, the Axie "bridge" now holds up to $\$ 600$ million in crypto assets, with some Axies worth up to 110 ETH (currently \$295,000 USD) [27]. Where the cost of attention is worth it, access to play and earn reputation and money is driving human behaviors within the algorithmic systems we design.

These algorithmic rules of governance are subjective. They are imbued with normative values and biases of their creator and the community in which they are formed. Algorithms convey a politics which the creator thinks should exist to determine what is and isn't desirable through incentives and rewards, including reputation, to define and shape algorithmic systems, and the societal systems subject to them. Technological systems are not neutral tools [28]. This subjectivity makes setting the rules of decentralized governance systems akin to the practice of policy making, in which algorithms perform and execute instructions in the way that they are encoded to do so to conduct governance functions [29]. Community norms are encoded in to the cryptoeconomic incentives that govern reputation in DAOs. They determine norms on ownership, access, incentives, labor, and rewards. When these decisions are taking in isolation, or with a short-term mindset, they can have adverse effects to incentivize undesirable behavior and produce negative consequences for the 
broader DAO, producing vulnerabilities instead of resilience to internal and external disruptions.

Algorithmic rules are difficult to define and DAOs are actively experimenting to do so. For example, reimbursement relates to incentivizing participation in DAOs, and whether people are volunteers, contractors, or stakeholders, and how they should be rewarded for their labor. If this is through pay, the DAO needs to determine how much, when, and whether this incentivizes nonvalues aligned, low-skill labor markets or a highly engaged, professionalized workforce. Among other DAO governance settings to be determined, token liquidity relates to whether a governance token should be liquid and tradeable, and if this will incentivize participation in governance, or if it will incentivize speculative markets and "protocol politics" rather than genuine, values-aligned contributors.

The idea of rule by dictatorship is antithetical to the community value of decentralization. Yet, in practice, a co-dependence exists between humans and machines in building algorithms to govern autonomous institutions. The social dynamics of having wellknown community members as decision makers in a DAO could be a strong social signal of legitimacy as people can trust known community members to make decisions in the best interests for stability of the DAO and towards its stated objective. Decentralized arbitration and accountability mechanisms in DAOs are also in their early days of experimentation. When a DAO is its own autonomous entity, there is no higher authority for further arbitration in the case of a dispute. If people are unsatisfied with the outcomes declared by Kleros or Aragon courts, has justice been served? "It becomes difficult to contend a decision when it is ostensibly made by a machine" warns Werbach, in relation to the SCS [30]. More-so, algorithmic rules must always be designed by someone.

Blockchainers often tout their enthusiasm in the freedom of choice presented in Hirchmann's idea of "exit, choice, and loyalty", the cost of exit can be high when it is your reputation, and coin [31]. Although people get to decide which communities they participate in and may even get to vote on which code upgrade gets accepted and which doesn't, the insidious monitoring of reputation in DAOs demonstrates how vulnerabilities can be natively introduced in DAOs to threaten the core values of these communities. The panopticon is a "cruel, ingenious cage" which does not even require constant observation by a central authority because decentralized peers will police each other out of their own fear and insecurity [32]. When participants design the system, who is working for who, and who is being made autonomous in autonomous systems?

\section{Case study: "Trust" score}

The Gitcoin project is a popular crowdsource funding platform that originated out of the Ethereum blockchain community to fund other projects. Gitcoin has recently transitioned to becoming a DAO by distributing digital tokens to past participants in the platform to become the "new bosses" of the system. To protect the platform against sybil attacks, Gitcoin employs a suite of identity verification tools that generate a reputational "trust score". Once registered on the platform, participants have an "activity score" that determines how active the user has been on the platform. High activity as well as good reviews and low levels of disputes determine how reliable a user is. Users are then incentivized to increase a "trust bonus" to qualify for a higher percentage of matched funds. This is achieved by linking the account with thirdparty decentralized identity providers or centralized social media platforms, such as Twitter and Facebook. Having a good reputation in the form of a high "trust score" perversifies behaviors as people are strongly incentivized to create multiple identities and stage sybil attacks to receive higher funding amounts.

The platform uses an algorithm known as "quadratic funding", which rewards people based on the number of donations they receive, rather than the value of donations, out of matched funding pools. Quadratic funding is when a crowdfunding campaign is correlated with a "matching pool" of funds, where rewards are calculated based on the number of people that support a campaign, rather than the amount donated [33]. It allows people or projects to donate to the matching pool, as a social signal of reputation and what they care about, and fundraisers to earn more if they garner wide support from the community. Quadratic funding has been heavily experimented with by the "Gitcoin" project, now GitcoinDAO, which allows projects in and around the Ethereum ecosystem to apply for funding from the community and contributes an important signal to the community of what is valuable and important. Whilst this function incentivizes large donations of matched funds as a social signal to support key areas of research and development in the Ethereum community, it also encourages micro donations, in the hope of some reward, meaning that project get hundreds of $\$ 1$ (equivalent in cryptocurrency) donations. This mechanism makes Gitcoin a lucrative platform to sybil attack to gain a higher percentage of matched funds. Grants round 9 in 2021 consisted of 168,000 donations, totaling $\$ 1.38$ million, which was matched by a further $\$ 500,000$ in funding [14]. Sybil attacks have increased per funding round as the financial rewards increase. Gitcoin's ability to function and grant donations in line with its stated objective and use the quadratic funding mechanism depends on the trust score mechanism to not being exploited by sybil attacks.

Recent rounds have been attacked by people that created bots to spam the platform with micro-donations so that they would receive a higher percentage of the pool of matching funds. There was also incentive to donate micro amounts to numerous projects in the hope of receiving a later reward of "Airdropped" tokes, as had occurred following the previous grants round [34]. A rapid governance intervention took place, and a machine learning pipeline was introduced to detect possible sybil behavior [14]. Now, an algorithm was designed and enacted as a technology to govern against sybil attacks by detecting and "flagging" 
algorithms attacking the network for having a suspicious reputation. It became a competition between algorithms and their human commanders about who would govern who between the sybil attackers and the sybil defenders. During this deployment, there was sharp debate amongst the Gitcoin team and key community stewards of the Machine Learning (ML) process over how much human involvement was appropriate, in what is meant to be a "decentralized", "autonomous" system. Some argued that the ML pipeline required human oversight to assess flagged activities and ensure that genuine users were not penalized by the algorithm. Other community members considered the algorithmic determination to be unbiased and argued against human intervention in the ML, stating the concern that people could retain "large amount[s] of power despite formal democratic oversight and control" by intervening in the algorithms put in place [14]. In the process of becoming a DAO and handing over core functions to community "stewards", the challenge arose for Gitcoin about how not to reveal this personally identifiable information but also "be decentralized". This surfaces tensions about whether an algorithm can truly be "decentralized" if designed and trained by people, and how much influence an algorithm should have over social and economic outcomes versus where human oversight is required. Now, community stewards of the "anti-sybil, anti-collusion" working group (renamed to "Fraud Detection \& Defense") were required to take responsibility for the critical function of anti-sybil in the DAO.

In this example, decentralized and algorithmic governance both mitigate against and introduce vulnerabilities in GitcoinDAO. With access to sensitive personal information, stewards were required to sign a non-disclosure agreement with Gitcoin the company, yet the simultaneous desire for the DAO to be decentralized complicates the priorities of how this function should be fulfilled effectively. Governing reputation and defending against sybil attacks is enhanced but not solved through algorithms. The project intends to approach the trust bonus from a "do no harm" standpoint. "How do we respect a user's privacy?" posed Gitcoin co-founder and CEO Kevin Owocki [35]. "Stated more forcefully: "Lets make sure we dont DOX our users", referring to an event whereby sensitive personal information is published on the internet [36]. In true hacker style, the project is responding by building things. A number of proposals are in flight to address this issue.

Another dynamic that influences institutional design and the role of algorithms to monitor and incentivize behavior in DAOs and the decentralized governance of critical organizational functions is scale. Once the system scales beyond the point when trust is based on direct social connections, people rely on either bureaucratic or algorithmic monitoring systems to enforce use behaviors to ensure responsibility, accountability, and recourse in the organization. Technical mechanisms to measure contributions are inherently mechanistic simplifications of the world. What can be quantified can be measured and what can be measured, can be rewarded to influence behavior in the game for trust between designers, algorithms, and participants.

Not just the risks but also the opportunities that algorithmic rules create are demonstrated in the matched quadratic funding mechanism of Gitcoin grants. Sometimes, algorithms inability to make certain judgements works in the favor of users.

The algorithmic rules surrounding reputation in Gitcoin can also unwittingly work to the advantage of participants. Reflecting on a recent Gitcoin funding round, Griff Green, a co-founder of "Commons Stack" (and a number of other long-standing projects in the community) described how a bounty that was posted many rounds ago for a Commons simulator game had automatically rolled over into the latest Gitcoin grants round. The project has several contributors based in Latin America which qualified it for the "Latin America" matched funding pool category. Although the project was basically on pause and some team based in Latin America was a loose definition of the funding category, the grant garnered the highest number of supporters for that category, giving it $\$ 35,000$, or $70 \%$, of the $\$ 50,000$ pool of matched funds. Meanwhile, this discrepancy in percentage of matched funds was made possible by the unspecified boundaries of the rules of the round and altering the algorithmic rules of the quadratic funding mechanism and wouldn't be credibly neutral. This reservedness to evaluate and adjust an algorithm decision-making system once functioning can benefit some, at the expense of others. Griff describes that "algorithmic governance is fucking me by giving me money right now!". Whilst it gave the team some explaining to do on how the project legitimately qualifies for the "Latin America" category, the algorithm did not discriminate. Of course, this was not necessarily to the benefit of the greater community, the goals of Gitcoin, or their own reputation, and they plan to pay the funds forward to the ecosystem. The algorithm was subjective and could be exploited for either individual gain, or have its outcomes opposed for collective benefit. System designers and decision makers were extremely hesitant to adjust the boundaries of the algorithm even when this situation was flagged, because they didn't want the algorithmic system, or human intervention in the system, to be seen as biased.

In these examples, we see algorithmic governance in blockchainbased ADMs working to both the benefit and risk of participants. Although these systems are under ongoing development and renegotiation, people can choose to use algorithms to shape the behavior of others to either subvert algorithmic rules or exploit algorithmic vulnerabilities, or to make them work in beneficial ways for the community.

\section{Analysis}

The question posed at the start of this piece was "Are "Decentralized Autonomous Organizations" the next social credit panopticon of algorithmic governance or a different panacea, and what does this mean for human autonomy in "autonomous" systems?'. The panopticon of algorithmic reputation monitoring 
has swiftly and quietly crept into the paradigm of Decentralized Autonomous Organizations, which were intended as a social institution that empowers participants as an alternative to the extractive digital infrastructures of corporate and government surveillance.

Part of living with our new algorithmic governors, is acknowledging they are there. As it stands, creators and participants oftentimes passively engage with algorithms in DAOs without acknowledging where and how they are operating. Transparency in terms of access to the raw code of an algorithm does not mean legibility, that people are able to understand how it works and effectively govern it, as it governs them [37]. Yet, transparency as to where algorithms are operating may be a first step in being able to critically analyse, question, and (heavenforbid) change the code towards more desirable settings for a long-term "win-win" game where people and algorithms adjust as needed, to continue to participate in the community towards a shared purpose. Participants in these institutions struggle with the dilemma of 'can we question code?' in what are meant to be immutable, post-human infrastructures. Some DAO communities are fostering a culture of consciously questioning the algorithms they want to govern them, and critically reflecting on the culture and values embedded in automated systems. This allows them to adapt in different circumstances, such as when sybil attackers develop new tactics, and collectively change the rules. Yet, cultivating and maintaining a culture of sensitivity to the explicit and tacit governance rules being encoded into algorithms may be a challenge for many DAOs as they scale.

DAOs are a particularly interesting site of algorithmic determination for internet communities as they attempt to offer decentralized institutional structures which may inform the future of online communities, data governance, and social coordination through digital means. DAOs are intended to be participatory digitally mediated institutions that reduce the need for social trust to empower participants to coordinate. In practice, algorithms at work in "decentralized" systems are not autonomous, in the same way that algorithms are not autonomous in existing digital infrastructure, and they afford enormous power to those who get to decide and encode the rules. Humans and algorithms are coconstitutive as they reflect the social, cultural, and political settings of their creators, and simultaneously provide incentives to dictate desired behaviors [38]. In "decentralized" digital platforms, it is a question of who gets to shape and influence the algorithms which in turn, govern the system. This affords great power and influence to DAO creators to determine the outcomes of others. In these infrastructural assemblages of code and people, the ability to consciously reflect on, shape, and iterate on the algorithmic governance rules of these experimental institutions in the practice of algorithmic policy making affords the people within them with adaptive capacity to participate in shaping the culture, goals, and operation of the system.
There is no such thing as autonomy in community. The need for subjective algorithms to monitor peoples' behaviors towards outcomes in "DAO" infrastructure shows that the idea of individual autonomy and self-governance fades in a trade-off with belonging to a collective community. Understandings of autonomy needs to be more deeply analyzed in the socio-technical imaginary of autonomous systems [39]. It is not yet clear whether it is the system being made autonomous, or the individuals within it. The two are inseparable, and some sense of autonomy must be sacrificed to coordinate as a collective. The question for DAO makers, and DAO participants becomes, what is given up at the individual level, for collective autonomy? The opportunity to collectively shape the rules of algorithmic governance creates both risks and new possibilities for these communities to define their own rules and adapt and evolve in response to these experiments and avoid perpetuating panopticon dynamics for the sake of algorithmic efficiency.

In the quest for autonomous assemblages, we see the machine working for the humans, and vice-versa. This co-dependence between human and machine shows that perhaps we are already "augmenting" our human capacities to coordinate with blockchain and smart contract infrastructure. The autonomy that is achieved in the interplay of algorithms and humans is a sense, even if an imaginary, of "self-governance" from external forces beyond the boundaries of the DAO, or the ability to shape the rules, and govern ourselves.

\section{Conclusion: trust, but verify}

The piece has explored DAOs and issues with algorithmic governance that could be exploited for originally unintended purposes. It has shown that algorithms are being readily experimented with in a participatory fashion for automated decision-making in blockchain-based systems to determine matters of reputation, access, resource allocation, and trust as a policymaking and society shaping practice, and how this could be emancipatory or dystopian.

I have outlines how DAOs are both an institutional structure for governance and a technology. Although Decentralized Autonomous Organizations aspire to provide new social coordination institutions, they can reinforce existing patterns of algorithmic exploitation seen in current internet infrastructures. Algorithms in blockchain-based reputation systems can easily be exploited to re-enforce algorithm authoritarianism in the same ways as existing digital infrastructures. Again, technology and automation are both an opportunity against the threat of external intervention, and a vulnerability in terms of what is promised versus what is created by "autonomous" algorithmic systems. Reputation systems form a fundamental component of the rules of governance, to determine how the game gets played and how algorithmic systems shape the behavior of participants. The question that designers need to ask themselves in how algorithmic 
rules are designed, encoded, and automated, is "who, or what, is being made autonomous, and at what cost or trade-off?".

In some cases, algorithms work to the advantage of participants and people acknowledge that the rules can be re-evaluated. The culture of some blockchain communities is that of intense curiosity and active experimentation, meaning that people can come together as a community to question the algorithm and improve it in line with the goals and values of a DAO. This reflexive practice within DAOs enables people to acknowledge their own biases and subjectivity and engage to help shape the culture that guides the rules being encoded into the system. These lessons are crucial to the design of governance in digital infrastructures.

This analysis has highlighted the exploitation of algorithmic governance in decentralized reputation systems as an emerging vulnerability in DAO governance, as well as an opportunity for decentralized technology communities to express their values and approach the design of digital infrastructure in accountable and participatory ways. Humans and algorithms are co-constitutive. The lessons from communities that can evaluate their algorithmic policies and maintain functional participation are to know where algorithms are operating, be able to question and re-evaluate them, and to lean on culture and objectives to guide the parameters of algorithmic governance. As humans and algorithms co-constitute one-another, the greatest threat to a DAO is not the external imposition of influence on its governance. The greatest threat to a DAO itself.

DAOs both address threats and vulnerabilities and create them, to both help and hinder resilience. Throughout sybil attacks, disagreements, and code changes, the people that participate in DAOs are resilient. In this way, the DAO itself is its greatest vulnerability is its greatest strength. The imaginary of "autonomy" as the ability to shape rules in DAO communities makes, maintains, and breaks a faithful following of participants. Processes for people and communities to actively engage to shape algorithmic rules is critical to resilience, as ongoing community participation in the face of disruptions. There is an incredible burden on engineers as the designers and decision makers of the algorithmic rules of these systems. Engineers have the power and responsibility in determining what kind of digital infrastructure is being created and whether this lives up to the imaginary of "autonomous" communities or deteriorates into the kind of control system that the Cypherpunks feared.

\section{ACKNOWLEDGMENTS}

With thanks to Professor Chris Berg, Professor Ellie Rennie, and Professor Sinclair Davidson for feedback. Thanks to Dr. Michael Zargham, Jeff Emmett, Burrrata, and David at Blockscience for ongoing research collaborations. Thank you to each person and community that engaged in interviews, including Gitcoin, Commons Stack, and the Token Engineering Commons.

\section{REFERENCES}

[1] Kelsie Nabben. 2021. "Experiments in algorithmic governance continue". Substack. Available online: https://kelsienabben.substack.com/p/experimentsin-algorithmic-governance, Accessed 5 August, 2021.

[2] Rishida Richardson. 2022 (Forthcoming). "Defining and Demystifying Automated Decision Systems". Maryland Law Review.

[3] Nick Szabo. 2017. "Money, blockchains, and social scalability", Unenumerated. 2017.2 Available online: https://unenumerated.blogspot.com/2017/02/money-blockchains-and-socialscalability.html. Accessed 18 August, 2021.

[4] S. Jasanoff \& S. Kim. 2015. Dreamscapes of Modernity: Sociotechnical Imaginaries and the Fabrication of Power. (University of Chicago Press), 6.

[5] B. Maurer, T.C. Nelms. \& L. Swartz. 2013. When perhaps the real problem is money itself! The practical materiality of Bitcoin. Social Semiotics, 23(2), 261277; Jan, Groos. "Crypto Politics: Notes on Sociotechnical Imaginaries of Governance in Blockchain Based Technologies." Data Loam, 148-170. De Gruyter, Astrid Mager, and Christian Katzenbach. 2020. "Future imaginaries in the making and governing of digital technology: Multiple, contested, commodified." New Media \& Society. (2021): 223-236. Doi: $10.1177 / 1461444820929321$.

[6] Jeremy Bentham. 1791. Panopticon Or the Inspection House, Volume 2. London.

[7] Shoshana Zuboff. 1989. In the Age of the Smart Machine: The Future of Work. (Basic Books).

[8] Vitalik Buterin. 2021. "Gitcoin Grants Round 9: The Next Phase of Growth Vitalik.ca. Available online: https://vitalik.ca/general/2021/04/02/round9.html. Accessed 20 June, 2021.

[9] Bo Zhao. 2015. "Reputation as Social Control in Present China: Use, Misuse, Abuse, and Bankruptcy". Asian Journal of Comparative Law. 10 (2). 359-379. doi:10.1017/asjcl.2015.16.

[10] J. R. Douceur. 2002. "The Sybil Attack". Peer-to-Peer Systems. Lecture Notes in Computer Science. pp. 251-260. Available online: https://archive.org/details/peertopeersystem0000iptp/page/251/mode/2up. Accessed 15 August, 2021.

[11] Jacob, Waterman. 2020. "Worldbuilding Politics and Power": Part 1 individuals. Nerdolopedia. Available online: https://www.nerdolopedia.com/articles/2020/1/9/worldbuilding-politics-andpower-part-1. Accessed 18 August, 2021.

[12] Adam, Levi. 2021. "Reputation vs Tokens". Medium. Available online: https://medium.com/daostack/reputation-vs-tokens-6d7642c7a538. Accessed 18 August, 2021; Vitalik, Buterin. 2021. "Moving beyond coin voting governance". Vitalik.ca. Available online: https://vitalik.ca/general/2021/08/16/voting3.html. Accessed 17 August, 2021.

[13] A. Sharma and A. Bhatia. 2020. "Bitcoin's Blockchain Data Analytics: A Graph Theoretic Perspective." arXiv preprint arXiv:2002.06403.

[14] Quinn, DuPont. 2017. "Experiment in algorithmic governance: A history and ethnography of "The DAO," a failed decentralized autonomous organization" in Bitcoin and Beyond. (Routledge). 171.

[15] J. Emmett, K. Nabben, D. L. Bernardineli, M. Zargham. 2021. "Deterring Adversarial Behaviour at Scale in Gitcoin Grants: a framework for communitybased algorithmic policy making". Medium. Available online: https://medium.com/block-science/deterring-adversarial-behavior-at-scale-ingitcoin-grants-a8a5cd7899ff. Accessed 1 August, 2021.

[16] T. C. May. 1988. "The Crypto Anarchist Manifesto". Available online: https://nakamotoinstitute.org/crypto-anarchist-manifesto/. Accessed 18 August, 2021.

[17] T. C. May. 1994. "Crypto Anarchy and Virtual Communities". Available online: https://nakamotoinstitute.org/virtual-communities/. Accessed 18 August, 2021.

[18] T. C. May. 1994/ "Virtual Commun.ties".

[19] Julie Cohen. 2019. Between Truth and Power: The Legal Constructions of Informational Capitalism. (Oxford University Press); Kate Klonick. 2017. "The New Governors: The People, Rules, and Processes Governing Online Speech' 131 Harv. L. Rev. 1598.

[20] Shoshana Zuboff. 2019. The Age of Surveillance Capitalism: The Fight for a Human Future at the New Frontier of Power. (Public Affairs).

[21] Kevin Werbach. 2021. "Panopticon Reborn: Social Credit as Regulation for the Algorithmic Age". (University of Illinois Law Review). Doi: 10.2139/ssrn.3589804. 28

[22] Kevin, Werbach. 2021. "Panopticon Reborn". 17

[23] Kate Crawford. 2021. Atlas of AI: Power, Politics, and the Planetary Costs of Artificial Intelligence. (Princeton University Press).

[24] Kelley Cotter. 2018. "Playing the visibility game: How digital influencers and algorithms negotiate influence on Instagram". New Media \& Society. 21: 4. 895-913. Doi: 10.1177/1461444818815684; José van Dijck and Thomas Poell. 2013. Understanding Social Media Logic. Media and Communication, 1(1) 214; Taina Bucher. 2012. "Want to be on top? Algorithmic power and the threat of invisibility on Facebook". New Media \& Society. 14(7). 1164-1180. 
[25] Leah Callon-Butler. 2021. "The NFT Game that Makes Cents for Filipinos During COVID”. CoinDesk. Available online: https://www.coindesk.com/nftgame-filipinos-covid. Accessed 10 August, 2021.

[26] Calvin Chu. 2021. "Impossible Rambles - Edition 1 | Calvin's Axie Infinity Obsession \& The Impossible Metaverse Thesis". Medium. Available online: https://medium.com/impossiblefinance/impossible-rambles-edition-1-calvinsaxie-infinity-obsession-the-impossible-metaverse-thesis-881a93a97c8a. Accessed 10 August, 2021

[27] Calvin Chu. 2021. "Impossible Rambles - Edition 1 | Calvin's Axie Infinity Obsession \& The Impossible Metaverse Thesis". Medium. Available onoline: https://medium.com/impossiblefinance/impossible-rambles-edition-1-calvinsaxie-infinity-obsession-the-impossible-metaverse-thesis-881a93a97c8a. Accessed October 20, 2021.

[28] Susan Leigh Star. 1999. The Ethnography of Infrastructure. American Behavioral Scientist 43, no. 3: 377-391. doi:10.1177/00027649921955326.

[29] Michael Zargham \& Kelsie Nabben. 2020. "Algorithms as Policy". Substack. Available online: https://kelsienabben.substack.com/p/algorithms-as-policy. Accessed August 18, 2021

[30] Werbach, K. "Panopticon Reborn". 2021. 41.

[31] A. O. Hirschmann. 1972. Exit, Voice, and Loyalty: Responses to decline in firms, organizations, and states. (Harvard University Press)

[32] Michel Foucault. 1977. "Discipline and Punish: The birth of the prison". (Alan Sheridan trans., Pantheon Books ed.). 222-27.

[33] Gitcoin. 2021. Gitcoin. "WTF is QF". Gitcoin.com. Available online: https://qf.gitcoin.co/?grant=\&grant=\&grant $=\&$ grant $=\&$ match $=1000 . \quad$ Accessed 01 August, 2021.

[34] Lefteris Karapetsas. 2021. A Tweet by @LefterisJP. Mar. 16, 2021. Available online: https://twitter.com/LefterisJP/status/1371474022450528261, Accessed 1 August, 2021; Vitalik Buterin. 2021. "Gitcoin Grants Round 9: The Next Phase of Growth. Vitalik.ca. Available online: https://vitalik.ca/general/2021/04/02/round9.html. Accessed 20 June, 2021.

[35] Kevin Owocki. 2021. Comments on "Establishing a New Process for Identity Verification Scoring (and removing troubled ID methods). Gitcoin Forum. Available online: https://gov.gitcoin.co/t/establishing-a-new-process-foridentify-verification-scoring-and-removing-troubled-id-methods/7506. Accessed 15 August, 2021.

[36] Kevin Owocki. 2021. Comments on "Establishing a New Process".

[37] Mike Ananny and Kate Crawford. 2018. "Seeing without knowing: Limitations of the transparency ideal and its application to algorithmic accountability." New Media \& Society 20: 973 - 989.

[38] Diana Forsythe. 2002. Studying Those Who Study Us: An Anthropologist in the World of Artificial Intelligence. (Stanford University Press).

[39] S. Jasanoff and S. Kim. 2015. Dreamscapes of Modernity". 